\title{
Superradiant phase transition with graphene embedded in one dimensional optical cavity
}

\author{
Benliang Li ${ }^{\text {a, b }}$, Tao Liu ${ }^{\text {a }}$, Daniel W. Hewak ${ }^{\text {b }}$, Qi Jie Wang a, c, * \\ a OPTIMUS, Centre for OptoElectronics and Biophotonics, School of Electrical \& Electronic Engineering, The Photonics Institute, Nanyang \\ Technological University, 50 Nanyang Ave, 639798, Singapore \\ b Optoelectronics Research Centre, University of Southampton, Southampton SO17 1BJ, United Kingdom \\ c CDPT, Centre for Disruptive Photonic Technologies, School of Physical and Mathematical Sciences, The Photonics Institute, Nanyang \\ Technological University, 637371, Singapore
}

\begin{abstract}
A B S T R A C T
We theoretically investigate the cavity QED of graphene embedded in an optical cavity under perpendicular magnetic field. We consider the coupling of cyclotron transition and a multimode cavity described by a multimode Dicke model. This model exhibits a superradiant quantum phase transition, which we describe exactly in an effective Hamiltonian approach. The complete excitation spectrum in both the normal phase and superradiant phase regimes is given. In contrast to the single mode case, multimode coupling of cavity photon and cyclotron transition can greatly reduce the critical vacuum Rabi frequency required for quantum phase transition, and dramatically enhance the superradiant emission by fast modulating the Hamiltonian. Our work paves a way to experimental explorations of quantum phase transitions in solid state systems.
\end{abstract}

\section{Introduction}

Cavity quantum electrodynamic (QED) describes the light-matter interaction in the quantum level which has been a fascinating topic. The excellent control and manipulation of the light-matter interaction can pave the way to fundamental studies and applications in quantum devices, such as plasmonic polaritons in graphene systems [1-3]. Recently, the physics of a collection of $N$ two-level atoms interacting with the same quantum field of a photonic mode described by celebrated Dicke model has stimulated great interests [4-13]. The Dicke model predicted a second-order quantum phase transition at particular coupling strength $\Omega_{c}$ in the $N \rightarrow \infty$ limit [14]. Such model allows us to investigate the superradiant (SR) phase of the system with a macroscopically excited and highly collective state that possesses the potential to be super-radiant. However, a more realistic model has to incorporate the quadratic field term $A^{2}(A$ is the vector potential of electromagnetic field) which was absent in the Dicke Hamiltonian. Meanwhile, it was shown that the quantum phase transition disappears in presence of the $A^{2}$ term as a consequence of the Thomas-Reiche-Kuhn (TRK) sum rule for the oscillator strength [15]. Therefore, systems such as cyclotron transition of two-dimensional electron gas, coupled with a cavity resonator and semiconductor intersubband transition coupled with a microcavity photon mode were shown to present large diamagnetic term $\left(A^{2}\right.$ term) which

\footnotetext{
* Corresponding author. Centre for OptoElectronics and Biophotonics, School of Electrical \& Electronic Engineering, The Photonics Institute, Nanyang Technological University, 50 Nanyang Ave, 639798, Singapore.

E-mail address: qjwang@ntu.edu.sg (Q.J. Wang).
} 
prevents quantum instability from happening [16], [17]. In contrast, two dimensional atomic materials such as graphene imbedded in an optical cavity resonator shows a superradiant quantum phase transition because of negligible $A^{2}$ term resulting from peculiar honeycomb structure of graphene [18], [19]. However, such systems are only described within the normal phase scope presently, the physical properties of graphene system beyond the quantum critical point have not yet been addressed. In this paper, we present a microscopic theory to describe the physics of graphene imbedded in a one dimensional (1D) optical cavity under a perpendicular magnetic field. We calculate the complete excitation spectrum in both the normal phase and superradiant phase regimes. The results show that multimode coupling of cavity photon and cyclotron transition, in contrast to the single mode case, can greatly reduce the critical vacuum Rabi frequency required for quantum phase transition, and dramatically enhance the superradiant emission by fast modulating the Hamiltonian. We want to emphasise that although the debate about graphene superradiant phase exits in single cavity mode case [18] [24], the conclusion can be robust via considering the multimode cavity modes in this paper [21].

The paper is organized as follows: In Section II, we develop the general theory of the coupling between a multimode optical cavity with cyclotron transition and establish the effective Hamiltonian below and above quantum critical point. In Section III, we discuss the excitation spectrum below and above quantum critical point. The superradiant quantum phase transitions in the cases of multimode and single cavity mode coupling are analysed. Finally, conclusions are drawn in Section IV. Additional theoretical derivations are presented in Appendices A-B.

\section{Physical system and effective Hamiltonian}

In the vicinity of the two inequivalent Dirac points $K$ and $K^{\prime}$, the low energy Hamiltonian can be written as $H_{L}=\hbar v_{F}\left(\zeta k_{x} \sigma_{x}+k_{y} \sigma_{y}\right)$ where $\sigma_{i}(i=x, y)$ are Pauli matrices, $v_{F} \approx 10^{6} \mathrm{~m} / \mathrm{s}$ is the Fermi velocity and $\zeta= \pm$ is the valley index. In this work, a static and uniform magnetic field $\boldsymbol{B}$ is applied along the $z$ axis perpendicularly to the graphene plane. As long as the lattice constant $a$ is much smaller than the magnetic length $l_{0}=\sqrt{\hbar /(e B)}$, we can perform the Landau-Peierls substitution which replace $\hbar \boldsymbol{k}$ with $\boldsymbol{\Pi}=\hbar \boldsymbol{k}+e \boldsymbol{A}_{0}$ where $\boldsymbol{A}_{0}(\boldsymbol{r})=(-B y / 2, B x / 2,0)$ is the vector potential in symmetric gauge. This yields the Landau level (LL) states in conduction band in $K$ and $K^{\prime}$ valleys as $\varphi_{n, l, K}^{\dagger}=(i\langle n-1, l|,\langle n, l|) / \sqrt{2}$ and $\varphi_{n, l, K^{\prime}}^{\dagger}=(\langle n, l|,-i\langle n-1, l|) / \sqrt{2}$ respectively where $n$ and $l$ are good quantum numbers of LL states in symmetric gauge, and the LL energies in conduction band as $E_{n}=\hbar \omega_{0} \sqrt{n}$ where $\omega_{0}=\sqrt{2} v_{F} / l_{0}$. Each LL has degeneracy $N=4 S /\left(2 \pi l_{0}^{2}\right)$ where $S$ represents the surface of the graphene layer. Note that here we take the spin and valley isospin degeneracy into account. We further define the LL filling factor as $v=\rho S / N+1 / 2$ [18] where $\rho$ is the electron doping density. In this paper, for the sake of simplicity, we consider the case of integer filling factor with the Fermi level being in the conduction band. As we deal with the coupling between the light and high filling factor of LLs, we have to take into account the system at cryogenic temperature. Therefore, we assume cryogenic temperature in the rest of the calculations of this paper to ensure the cyclotron transition energy is larger than thermal energy. The loss of graphene will not influence the excitation of spectrum of polariton. But large loss will prevent from observing the spectrum of polariton. The considered cryogenic temperature can ensure the loss smaller than Rabi frequency in our considered parameters.

In this work, we consider 1D optical cavity $\left(L_{x} \gg L_{z} \gg L_{y}\right)$ with graphene monolayer placed in the middle at $z=L_{z} / 2$ as depicted in Fig. 1. This permits us to treat the continuous dispersion along the $x$ axis while keeping a few modes along $z$ axis and neglecting all the modes along the $y$ axis except the lowest one. The vector potential $\boldsymbol{A}_{e m}(\boldsymbol{r})$ of cavity field can be written as

$$
\boldsymbol{A}_{\boldsymbol{e m}}(\boldsymbol{r})=\sum_{\boldsymbol{q}} \sqrt{\frac{\hbar}{2 \varepsilon_{0} \varepsilon \omega_{\boldsymbol{q}}}}\left(u_{\boldsymbol{q}} a_{\boldsymbol{q}}+u_{\boldsymbol{q}}^{*} a_{\boldsymbol{q}}^{\dagger}\right)
$$

where $\varepsilon$ is the cavity dielectric constant and operators $a_{\boldsymbol{q}}$ are the photon annihilation operators with corresponding wave vector $\boldsymbol{q}$ written as $\boldsymbol{q}=\left(2 \pi n_{x} / L_{x}, 0, \pi n_{z} / L_{z}\right)$, the mode spatial profile $u_{\boldsymbol{q}}$ reads

$$
u_{\boldsymbol{q}}=\sqrt{\frac{2}{V}} \exp \left(i \frac{2 \pi n_{x} x}{L_{x}}\right) \sin \left(\frac{\pi n_{z}}{2}\right)(0,1,0)
$$

Note that only the photonic modes with $n_{z}$ being the odd integers will be coupled to the LL transitions. The cavity modes frequencies are given as

$$
\omega_{\boldsymbol{q}}=\frac{c}{\sqrt{\varepsilon}} \sqrt{\left(\frac{\pi n_{z}}{L_{z}}\right)^{2}+\left(\frac{2 \pi n_{x}}{L_{x}}\right)^{2}}
$$

The interaction Hamiltonian for the cavity-graphene system is given as $H_{\text {int }}=e v_{F} \boldsymbol{A}_{e m} \cdot \boldsymbol{\sigma}$. Here we investigate the coupling between the cavity modes and the cyclotron transition between the last occupied $\operatorname{LL} n=v-1$ and the first unoccupied one $n=v$, and the second quantization form of interaction Hamiltonian reads (see Appendix A) 


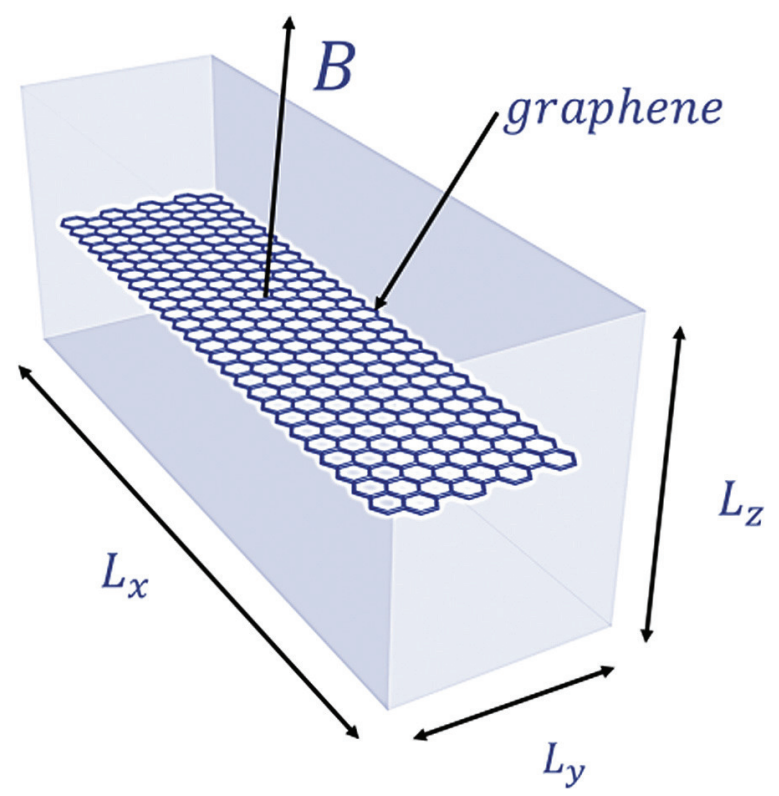

Fig. 1. Sketch of a one dimensional cavity embedding graphene sheet, a uniform and static magnetic field $B$ is applied along the $z$ axis, more information of such 1D cavity can be found in Ref. [17].

$$
\frac{H_{\text {int }}}{\hbar}=\sum_{\boldsymbol{q}, l} \frac{\Omega_{\boldsymbol{q}}}{\sqrt{N}}\left(a_{\boldsymbol{q}}^{\dagger}+a_{\boldsymbol{q}}\right)\left(c_{v, l}^{\dagger} c_{v-1, l}+c_{v-1, l}^{\dagger} c_{v, l}\right)
$$

where the vacuum Rabi frequency $\Omega_{\boldsymbol{q}}=\frac{e v_{F}}{2} \sqrt{\frac{N}{\varepsilon_{0} \varepsilon \omega_{\boldsymbol{q}} V \hbar}}$ and $V$ being the cavity volume.

In order to bosonize the LL band excitations, we apply the Hosltein-Primakoff representation [20], where the effective Hamiltonian associated to the system reads

$$
\frac{H}{\hbar}=\omega_{\mathrm{eg}} b^{\dagger} b+\sum_{\boldsymbol{q}} \omega_{\boldsymbol{q}} a_{\boldsymbol{q}}^{\dagger} a_{\boldsymbol{q}}+\sum_{\boldsymbol{q}} \frac{\Omega_{\boldsymbol{q}}}{\sqrt{N}}\left(a_{\boldsymbol{q}}^{\dagger}+a_{\boldsymbol{q}}\right)\left(b^{\dagger} \sqrt{N-b^{\dagger} b}+\sqrt{N-b^{\dagger} b} b\right)
$$

where $\omega_{e g} \equiv \omega_{0}(\sqrt{v}-\sqrt{v-1})$ being the transition frequency between two nearby LLs.

This is a multimode Dicke model which can be solved both in normal and SR phases [21]. Below the critical point (normal phase), the effective Hamiltonian in normal phase is obtained as

$$
\frac{H_{\mathrm{No}}}{\hbar}=\omega_{\mathrm{eg}} b^{\dagger} b+\sum_{\boldsymbol{q}} \omega_{\boldsymbol{q}} a_{\boldsymbol{q}}^{\dagger} a_{\boldsymbol{q}}+\sum_{\boldsymbol{q}} \Omega_{\boldsymbol{q}}\left(a_{\boldsymbol{q}}^{\dagger}+a_{\boldsymbol{q}}\right)\left(b^{\dagger}+b\right)
$$

Then the excitation spectrum of $\omega_{j}$ is given by solutions to the equation as

$$
4 \omega_{e g} \sum_{\boldsymbol{q}} \frac{\omega_{\boldsymbol{q}} \Omega_{\boldsymbol{q}}^{2}}{\omega_{\boldsymbol{q}}^{2}-\omega_{j}^{2}}=\omega_{e g}^{2}-\omega_{j}^{2}
$$

Note that the excitation energies $\omega_{j}$ are real only for $\eta \equiv \gamma_{c} / \gamma>1$ where $\gamma \equiv \sum_{\boldsymbol{q}} \Omega_{\boldsymbol{q}}^{2} / \omega_{\boldsymbol{q}}$ and $\gamma_{c} \equiv \omega_{e g} / 4$. The value of $\eta=1$ separates the normal and SR phases. The summation of all the coupling photonic modes in $\gamma$ shows that critical Rabi frequency required for quantum phase transition can be reduced by coupling matter to a multimode cavity compared with single cavity mode case.

Above the critical point $(\eta<1)$, we displace the Bosonic modes in Eq. (5) by $\tilde{a}_{\boldsymbol{q}}=a_{\boldsymbol{q} \pm \sqrt{\alpha_{\boldsymbol{q}}}}$ and $\tilde{b}=b \mp \sqrt{\beta}$, and eliminate the terms in Eq. (5) that are linear in the Bosonic operators by choosing $\beta=\frac{N}{2}(1-\eta)$ and $\alpha_{\boldsymbol{q}}=\frac{4 \beta(N-\beta) Q_{q}^{2}}{N \omega_{q}^{2}}$. Note that we obtain exactly the same values of $\alpha_{\boldsymbol{q}}$ and $\beta$ regardless of which sign of the operator displacements we choose. The effective Hamiltonian above the phase transition point becomes 


$$
\frac{H_{\mathrm{SR}}}{\hbar}=\sum_{\boldsymbol{q}} \omega_{\boldsymbol{q}} \tilde{a}_{\boldsymbol{q}}^{\dagger} \tilde{a}_{\boldsymbol{q}}+\tilde{\omega}_{\mathrm{eg}} \tilde{b}^{\dagger} \tilde{b}+\sum_{\boldsymbol{q}} \tilde{\Omega}_{\boldsymbol{q}}\left(\tilde{a}_{\boldsymbol{q}}^{\dagger}+\tilde{a}_{\boldsymbol{q}}\right)\left(\tilde{b}^{\dagger}+\tilde{b}\right)+f\left(\tilde{b}^{\dagger 2}+\tilde{b}^{2}\right)+\text { const }
$$

where $\tilde{\omega}_{\mathrm{eg}}=\frac{\omega_{\mathrm{eg}}\left(-\eta^{2}+6 \eta+3\right)}{4 \eta(\eta+1)}, \tilde{\Omega}_{\boldsymbol{q}}=\Omega_{\boldsymbol{q}} \eta \sqrt{\frac{2}{1+\eta}}$ and $f=\frac{\omega_{\mathrm{eg}}\left(-3 \eta^{2}+2 \eta+1\right)}{8 \eta(\eta+1)}$.

Then the excitation spectrum $\tilde{\omega}_{j}$ above the critical point is given as

$$
4\left(\tilde{\omega}_{e g}-2 f\right) \sum_{\boldsymbol{q}} \frac{\omega_{\boldsymbol{q}} \tilde{\Omega}_{\boldsymbol{q}}^{2}}{\omega_{\boldsymbol{q}}^{2}-\tilde{\omega}_{j}^{2}}=\tilde{\omega}_{e g}^{2}-4 f^{2}-\tilde{\omega}_{j}^{2}
$$

Note that beyond the transition point, the parity symmetry of the system has become spontaneously broken. In normal phase, the parity operator reads $\Pi_{\mathrm{No}}=\exp \left[i \pi\left(\boldsymbol{b}^{\dagger} b+\sum_{\boldsymbol{q}} a_{\boldsymbol{q}}^{\dagger} a_{\boldsymbol{q}}\right)\right]$ and it commutes with normal phase Hamiltonian $\left[\Pi_{\mathrm{No}}, H_{\mathrm{No}}\right]=$ 0 satisfying parity symmetry. In SR phase, parity symmetry is broken $\left[\Pi_{\mathrm{No}}, H_{\mathrm{SR}}\right]=0$, and system obeys a new parity symmetry $\left[\Pi_{\mathrm{SR}}, H_{\mathrm{SR}}\right]=0$ where $\Pi_{\mathrm{SR}}=\exp \left[i \pi\left(\tilde{b}^{\dagger} \tilde{b}+\sum_{\mathbf{q}} \tilde{a}_{\mathbf{q}}^{\dagger} \tilde{a}_{\boldsymbol{q}}\right)\right]$.

\section{Results and discussions}

In Fig. 2(a) and (b), we show the complete spectrum of the magnetopolariton both in normal and SR phases as a function of doping density $\rho$ and magnetic field strength $B$ respectively. In Fig. 2 (a), the SR phase transition occurs at $\rho_{\mathrm{c}}=2.42 \times 10^{9} \mathrm{~cm}^{-2}$
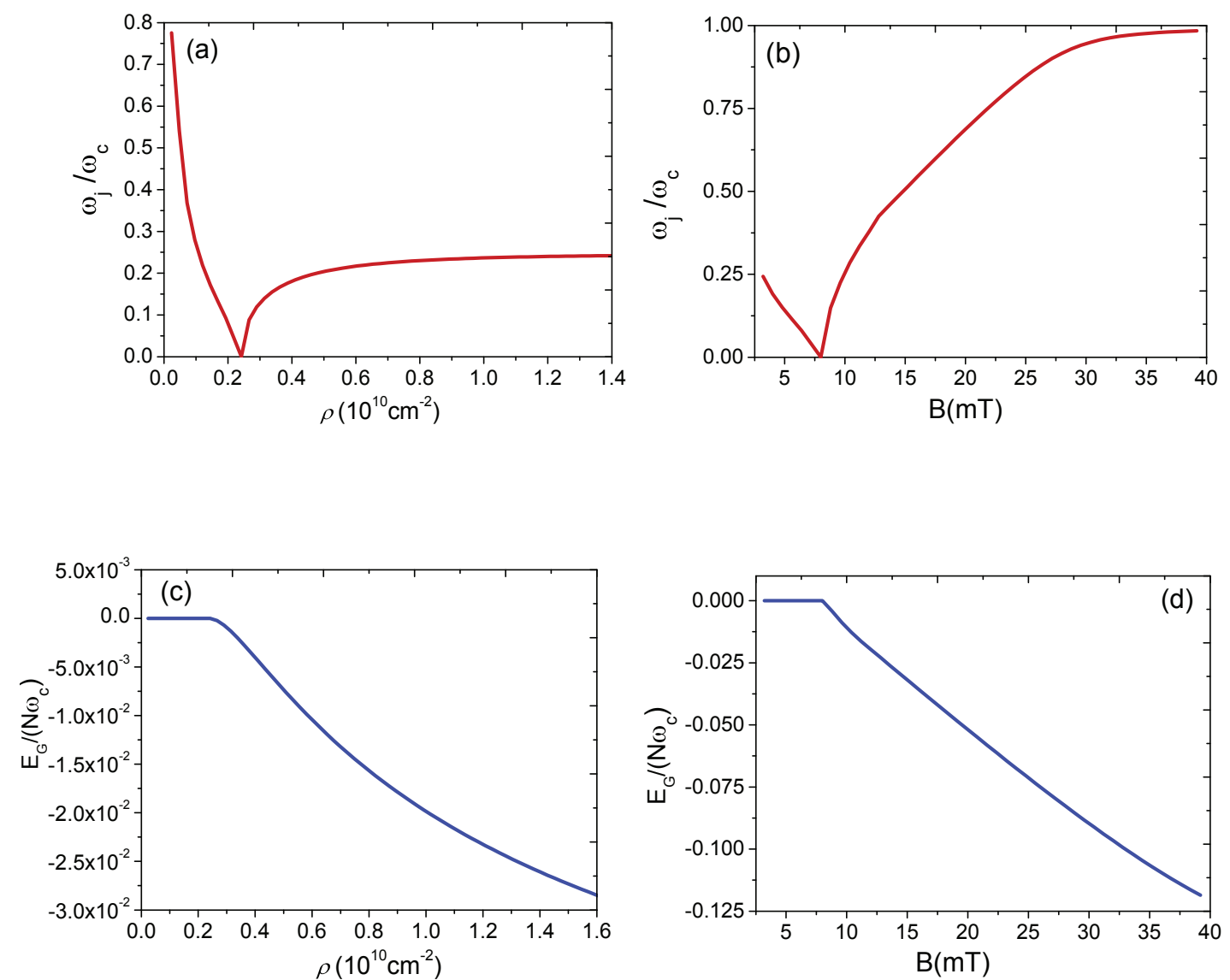

Fig. 2. (a) Normalized frequencies of magnetopolariton both in normal and SR phases as a function of doping density $\rho$ with $B=8 m T$ and critical density for phase transition is $\rho_{\mathrm{c}}=2.42 \times 10^{9} \mathrm{~cm}^{-2}$. (b) Normalized frequencies of magnetopolariton both in normal and SR phases as a function of magnetic field B with doping density $\rho=2.40 \times 10^{9} \mathrm{~cm}^{-2}$ and the critical value of magnetic field for phase transition is $B_{c}=8 \mathrm{mT}$. (c) The scaled ground state energy both in normal and SR phases as a function of doping density $\rho$ with $B=8 \mathrm{mT}$. (d) The scaled ground state energy both in normal and SR phases as a function of magnetic field B with doping density $\rho=2.40 \times 10^{9} \mathrm{~cm}^{-2}$. The rest parameters are $L_{z}=100 \mu \mathrm{m}, L_{x}=25 L_{z}, L_{y}=L_{z} / 5$ and $\omega_{c}=4.7 \mathrm{THz}$ being the lowest lying cavity mode frequency. With these parameters, only one branch of magnetopolaritons shows up (as shown in Fig. 2. (a)-(b)) and this result is obtained with a cutoff value of $n_{z}<7$. 


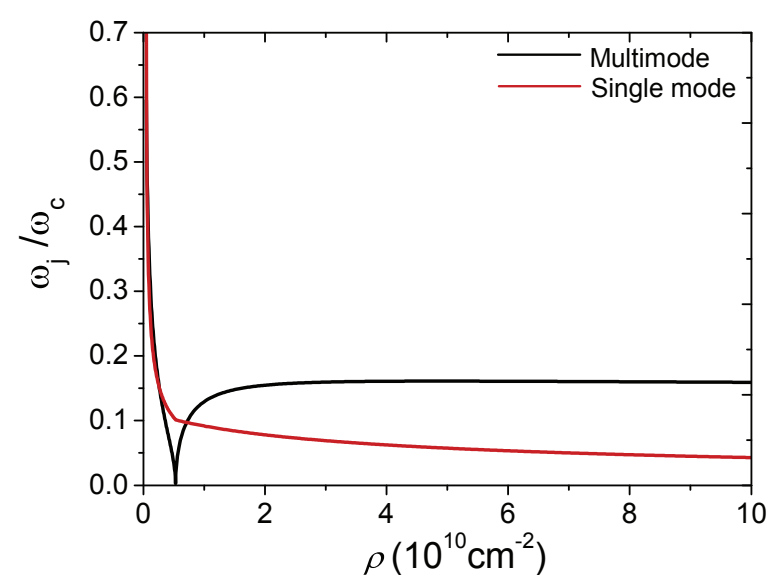

Fig. 3. Normalized frequencies of magnetopolariton for multimode versus single-mode as a function of doping density $\rho$. Parameters for multimode case are $B=8 m T, L_{z}=100 \mu m, L_{x}=25 L_{z}, L_{y}=L_{z} / 5$ and $\omega_{c}=4.7 \mathrm{THz}$ being the lowest lying cavity mode frequency. For the single mode case, the photonic mode frequency $\omega=\omega_{c}=4.7 \mathrm{THz}$. The critical density for phase transition in the multimode case is $\rho_{\mathrm{c}}=5.32 \times 10^{9} \mathrm{~cm}^{-2}$, this result is obtained with $n_{z}=1$. Note that the critical density for the single mode phase transition is $\rho_{\mathrm{c}}=4.38 \times 10^{11} \mathrm{~cm}^{-2}$ which is around 100 times larger than the critical density in multi-mode case.
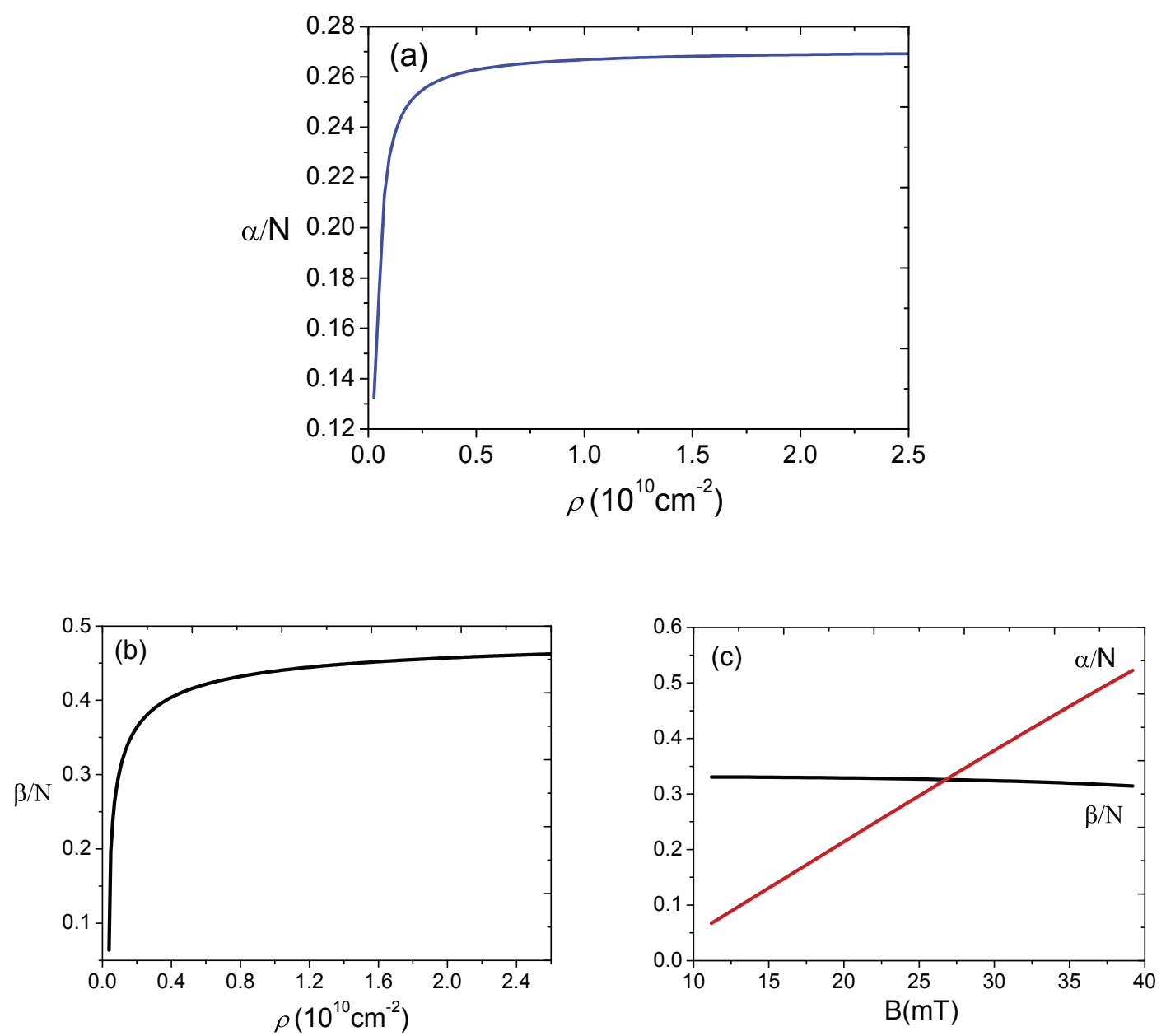

Fig. 4. (a) The scaled mean photon number as a function of doping density $\rho$ with magnetic field $B=8 m T$. (b) The electronic inversion as a function of doping density $\rho$ with magnetic field $B=8 m T$. (c) The scaled mean photon number and the electronic inversion as a function of the magnetic field B with doping density $\rho=2.40 \times 10^{9} \mathrm{~cm}^{-2}$. The rest parameters are $L_{z}=200 \mu m, L_{x}=50 L_{z}$ and $L_{y}=L_{z} / 5$. 


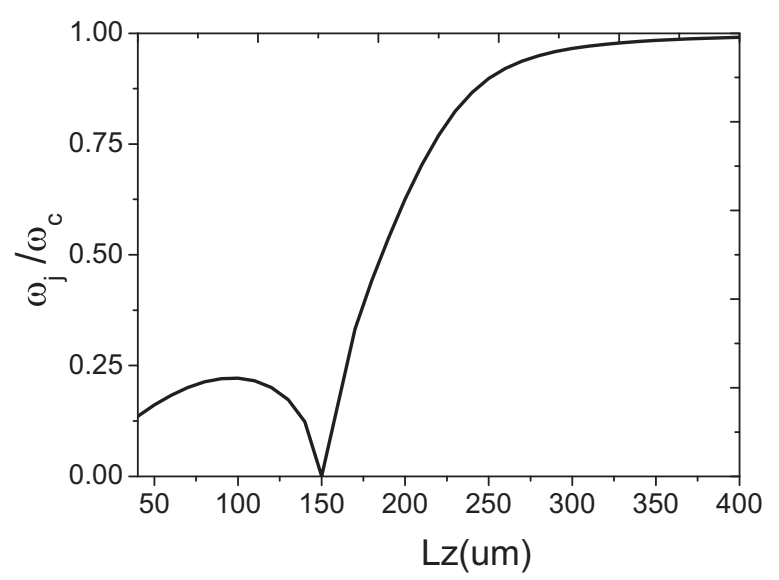

Fig. 5. Normalized frequencies of magnetopolariton both in normal and SR phases as a function of cavity dimension $L_{z}$ with $B=8 m T$ and the doping density is $\rho_{c}=1.06 \times 10^{9} \mathrm{~cm}^{-2}$. The critical value of cavity dimensional for phase transition is $L_{z}=150 \mu \mathrm{m}$. The other parameters are $L_{x}=25 L_{z}, L_{y}=L_{z} / 5$.

and in Fig. 2(b), the SR phase transition occurs at $B=8 m T$ with our considered parameters. By analysing the behaviour of the excited state energy relatively to the ground state in the vicinity of the critical point, we find $\omega_{j} \sim\left|\rho-\rho_{c}\right|^{z v}$, here $z v=1 / 2$ is the critical exponent, the first derivative of the excited state energy with respect to the doping density is $\frac{d \omega_{j}}{d \rho} \sim\left|\rho-\rho_{c}\right|^{-1 / 2}$ which diverges at the critical value, this marks the second order quantum phase transition. Meanwhile, we calculate the scaled ground state energy as $E_{G} /\left(N \omega_{c}\right)=-(1-\eta)^{2} \hbar \omega_{e g} /\left(4 \eta \omega_{c}\right)$ shown in Fig. 2(c) and (d) where we scale this quantity by $N$ followed by normalization with respect to the lowest lying cavity mode frequency $\omega_{c} \equiv c \pi /\left(L_{z} \sqrt{\varepsilon}\right)$. As we can see in Fig. 2(c) and (d), the system indeed shows a qualitative change in the ground state at the phase transition point beyond which the system tends to stabilize itself into the SR phase.

Fig. 3 shows the magnetopolariton frequencies calculated from the cases of multiple cavity modes coupling and only single mode coupling as function of doping density. The result shows that the critical doping density required for SR phase transition is much smaller in multiple modes coupling than that in single mode coupling. This implies that we should consider a multimode cavity to experimentally observe the SR phase transition.

In Fig. 4, we plot scaled mean photon number $\alpha=\sum_{\boldsymbol{q}}\left\langle a_{\boldsymbol{q}}^{\dagger} a_{\boldsymbol{q}}\right\rangle$ and electronic inversion $\left\langle b^{\dagger} b\right\rangle=\beta$. This figure clearly illustrates that, in the normal phase, the system is only microscopically excited, whereas above a certain critical doping density both the field and electronic ensemble acquire macroscopic excitations. In Fig. 4 (c), since the degeneracy $N=4 S /\left(2 \pi l_{0}^{2}\right)$ has a linear dependence of the magnetic field $\mathrm{B}$, therefore, we get the nearly linear dependence of the scaled quantities $\alpha$ and $\beta$. Noted that photons in the ground state of SR regime are virtual and they cannot be escaped from the cavity in the case of timeindependent Hamiltonians [23]. So multimode coupling of cavity photon and cyclotron transition can dramatically enhance the superradiant emission if we fast modulate the Hamiltonian compared with single mode case. For Fig. 5, we plot the normalized magnetopolariton frequency versus the cavity thickness $L_{z} \cdot \omega_{c} \equiv c \pi /\left(L_{z} \sqrt{\varepsilon}\right)$ is the lowest cavity mode frequency which depends on the cavity thickness $L_{z}$. Note that for large cavities, the frequencies of the cavity modes become continuous, in such scenario, we cannot discard some higher order modes frequencies when we calculate the magnetopolariton frequencies. Therefore, the results become inaccurate for larger cavities. Fig. 5 is plotted with a cutoff range with $L_{z}<400 \mu m$.

\section{Conclusions}

In conclusion, we theoretically present the excitation spectrum of graphene embedded in an optical cavity under perpendicular magnetic field. We consider the coupling of cyclotron transition and a multimode cavity described by a multimode Dicke model. The model predicts a superradiant phase transition will occur in graphene while such quantum phase transition does not exist in conventional semiconductors. The complete excitation spectrum in both the normal phase and superradiant phase regimes is given. In contrast to the single mode case, multimode coupling of cavity photon and cyclotron transition can greatly reduce the critical vacuum Rabi frequency required for quantum phase transition, and dramatically enhance the superradiant emission by fast modulating the Hamiltonian.

\section{Appendix A. . Second quantization of interaction Hamiltonian}

As we mentioned before, the interaction Hamiltonian can be written as $H_{\mathrm{int}}=e v_{\mathrm{F}} \boldsymbol{A}_{e m} \cdot \boldsymbol{\sigma}$, projecting this Hamiltonian in LL basics of conduction band, we obtain 


$$
H_{\mathrm{int}}=\sum_{n, l, n^{\prime}, l^{\prime}} \frac{e v_{F}}{2}\left(\left\langle n, l\left|\boldsymbol{A}_{\boldsymbol{e m}}\right| n^{\prime}-1, l^{\prime}\right\rangle+\left\langle n-1, l\left|\boldsymbol{A}_{\boldsymbol{e m}}\right| n^{\prime}, l^{\prime}\right\rangle\right) c_{n, l}^{\dagger} c_{n^{\prime}, l^{\prime}}
$$

where $\boldsymbol{A}_{\boldsymbol{e m}}$ is given in Eq. (1). Bearing in mind that we are dealing with the cyclotron transitions between the last occupied LL $n=v-1$ with the first unoccupied one $n=v$, therefore the $H_{\text {int }}$ can be simplified as

$$
H_{\mathrm{int}}=\sum_{l, l^{\prime}} \frac{e v_{F}}{2}\left\langle v-1, l\left|\boldsymbol{A}_{\boldsymbol{e m}}\right| v-1, l^{\prime}\right\rangle\left(c_{v, l}^{\dagger} c_{v-1, l^{\prime}}+c_{v-1, l^{\dagger}}^{\dagger} c_{v, l^{\prime}}\right)
$$

Since we are dealing with low energy cavity modes, the LL mixing can be neglected [22] and we apply the formula

$$
\left\langle n, l|\exp (-i \boldsymbol{q} \cdot \boldsymbol{r})| n^{\prime}, l^{\prime}\right\rangle=\exp \left(-\mid \mathbf{q}^{2} l_{0}^{2} / 2\right) \chi_{n, n^{\prime}}\left(q l_{0}\right) \chi_{l, l^{\prime}}\left(-q^{*} l_{0}\right)
$$

where $\chi_{n, n^{\prime}}\left(q l_{0}\right) \equiv \Theta\left(n-n^{\prime}\right) G_{n, n^{\prime}}\left(q^{*} l_{0}\right)+\Theta\left(n^{\prime}-n\right) G_{n^{\prime}, n}\left(q l_{0}\right)$ and

$$
G_{n, n^{\prime}}\left(q l_{0}\right)=\sqrt{\frac{n^{\prime} !}{n !}}\left(\frac{-i q l_{0}}{\sqrt{2}}\right)^{n-n^{\prime}} \sum_{j=0}^{n^{\prime}} \frac{n !}{\left(n^{\prime}-j\right) !\left(n-n^{\prime}+j\right) !} \frac{\left(-\left|q l_{0}\right|^{2}\right)^{j}}{2^{j j !}}
$$

Note that $q=q_{x}+i q_{y}$ and $\Theta(n)$ is Heaviside step function. Thus we have $\left\langle v-1, l\left|\exp \left( \pm i q_{x} x\right)\right| v-1, l^{\prime}\right\rangle=\delta_{l, l^{\prime}}$ thanks to this condition $|\boldsymbol{q}| l_{0} \ll 1$. Finally we arrive at

$$
\frac{H_{\mathrm{int}}}{\hbar}=\sum_{\boldsymbol{q}, l} \frac{\Omega_{\boldsymbol{q}}}{\sqrt{N}} \sin \left(\frac{\pi n_{3}}{2}\right)\left(a_{\boldsymbol{q}}^{\dagger}+a_{\boldsymbol{q}}\right)\left(c_{v, l}^{\dagger} c_{v-1, l}+c_{v-1, l}^{\dagger} c_{v, l}\right)
$$

We notice that only cavity modes with $n_{3}$ being odd number are coupled with LL transitions. At last, $\sin \left(\pi n_{3} / 2\right)$ can be absorbed in photon creation and annihilation operators by transformation $\sin \left(\pi n_{3} / 2\right)\left(a_{\boldsymbol{q}}+a_{\boldsymbol{q}}^{\dagger}\right) \rightarrow\left(a_{\boldsymbol{q}}+a_{\boldsymbol{q}}^{\dagger}\right)$ due to the fact that $\sin \left(\frac{\pi n_{3}}{2}\right)=1$ or $\sin \left(\frac{\pi n_{3}}{2}\right)=-1$.

\section{Appendix B. . The spectrum of magnetopolaritons}

As we mentioned before, Eq. (7) together with Eq. (9) gives the complete spectrum of the magnetopolaritons in the system for both normal and SR phases. The condition $L_{x} \gg L_{z}$ allows us to make replacement: $\sum_{\boldsymbol{q}} \rightarrow \sum_{n_{z}} \int g_{n_{z}}(\omega) d \omega$ where

$$
g_{n_{z}}(\omega)=\frac{\omega \varepsilon L_{x}}{\pi c^{2} \sqrt{\frac{\omega^{2} \varepsilon}{c^{2}}-\left(\frac{n_{z} \pi}{L_{z}}\right)^{2}}}
$$

being the density of the modes, therefore Eq. (9) becomes

$$
\frac{e^{2} v_{F}^{2} \omega_{e g} L_{x} N \eta}{2 \pi c^{2} \varepsilon_{0} \hbar V} \sum_{n_{z}} \int_{0}^{\infty} \frac{d y}{y^{2}+u_{n_{z}}}=\frac{\omega_{e g}^{2}\left(-\eta^{2}+2 \eta+1\right)}{2 \eta^{2}}-\tilde{\omega}_{j}^{2}
$$

where $u_{n_{z}} \equiv \frac{n_{z}^{2} \pi^{2}}{L_{z}^{2}}-\frac{\varepsilon \tilde{\omega}_{j}^{2}}{c^{2}}$ and $y \equiv \sqrt{\frac{\omega^{2} \varepsilon}{c^{2}}-\left(\frac{n_{z} \pi}{L_{z}}\right)^{2}}$. In order to perform the integration in the left hand side of Eq. (B2), we consider the situation $u_{n_{z}=1}>0$, therefore we have $\int_{0}^{\infty} \frac{d y}{y^{2}+u_{n_{z}}}=\frac{\pi}{2 \sqrt{u_{n_{z}}}}$, thus the spectrum of magnetopolaritons can be obtained under condition $u_{n_{2}=1}>0$ which being plotted in Fig. 2(a).

The same technique applies in the calculations of magnetopolaritons in normal phase.

\section{References}

[1] Y. Kim, S. Yu, N. Park, Low-dimensional gap plasmons for enhanced light-graphene interactions, Sci. Rep. 7 (2017) 43333.

[2] H. Lu, X. Gan, B. Jia, D. Mao, J. Zhao, Tunable high-efficiency light absorption of monolayer graphene via Tamm plasmon polaritons, Opt. Lett. 41 (20) (2016) 4743-4746.

[3] H. Lu, Plasmonic characteristics in nanoscale graphene resonator-coupled waveguides, Appl. Phys. B 118 (2015) 61-67.

[4] R.H. Dicke, Coherence in spontaneous radiation processes, Phys. Rev. 93 (1954) 99-110.

[5] K. Hepp, E.H. Lieb, On the superradiant phase transition for molecules in a quantized radiation field: the dicke master model, Ann. Phys. 76 (1973) $360-404$. 
[6] F.T. Hioe, Phase transitions in some generalized dicke models of superradiance, Phys. Rev. A 8 (1973) 1440-1445.

[7] G.C. Duncan, Effect of antiresonant atom-field interactions on phase transitions in the Dicke model, Phys. Rev. A 9 (1974) $418-421$.

[8] K. Hepp, E.H. Lieb, Equilibrium statistical mechanics of matter interacting with the quantized radiation field, Phys. Rev. A 8 (1973) $2517-2525$.

[9] V.I. Emeljanov, Y.L. Klimontovich, Appearance of collective polarization as a result of phase transition in an ensemble of two-level atoms, interacting through electromagnetic field, Phys. Lett. A 59 (1976) 366-368.

[10] K. Rzażewski, K. Wódkiewicz, W. Zakowicz, Phase transitions, two-level atoms, and the A2 term, Phys. Rev. Lett. 35 (1975) $432-434$.

[11] K. Rzażewski, K. Wódkiewicz, Thermodynamics of two-level atoms interacting with the continuum of electromagnetic field modes, Phys. Rev. A 13 (1976) 1967-1969.

[12] B.V. Thompson, A canonical transformation theory of the generalized Dicke model, J. Phys. A Math. General 10 (1977) 89-97.

[13] B.V. Thompson, Soft modes and the counter-rotating terms of the Dicke model, J. Phys. A Math. General 10 (1977) L179-L182.

[14] C. Emary, T. Brandes, Chaos and the quantum phase transition in the Dicke model, Phys. Rev. E 67 (066203) (2003).

[15] P. Nataf, C. Ciuti, No-go theorem for superradiant quantum phase transitions in cavity QED and counter- example in circuit QED, Nat. Commun. 1 (2010) 72 .

[16] C. Ciuti, G. Bastard, I. Carusotto, Quantum vacuum properties of the intersubband cavity polariton field, Phys. Rev. B 72 (2005), 115303.

[17] D. Hagenmuller, S.D. Liberato, C. Ciuti, Ultra strong coupling between a cavity resonator and the cyclotron transition of a two dimensional electron gas in the case of an integer filling factor, Phys. Rev. B 81 (2010), 235303.

[18] D. Hagenmuller, C. Ciuti, Cavity QED of the graphene cyclotron transition, Phys. Rev. Lett. 109 (2012), 267403.

[19] T. Liu, Q.J. Wang, Magnetopolariton in bilayer graphene: a tunable ultrastrong light-matter coupling, Phys. Rev. B 89 (2014), 125306.

[20] T. Holstein, H. Primakoff, Field dependence of the intrinsic domain magnetization of a ferromagnet, Phys. Rev. 58 (1949) 1098.

[21] D. Tolkunov, D. Solenov, Quantum phase transition in the multimode Dicke model, Phys. Rev. B 75 (024402) (2007).

[22] R. Roldan, J.N. Fuchs, M.O. Goerbig, Spin-flip excitations, spin waves, and magnetoexcitons in graphene Landau levels at integer filling factors, Phys. Rev. B 82 (2010), 205418.

[23] S.D. Liberato, C. Ciuti, I. Carusotto, Quantum vacuum radiation spectra from a semiconductor microcavity with a time-modulated vacuum Rabi frequency, Phys. Rev. Lett. 98 (2007), 103602.

[24] L. Chirolli, M. Polini, V. Giovannetti, A.H. MacDonald, DrudeWeight, cyclotron resonance, and the dicke model of graphene cavity QED, Phys. Rev. Lett. 109 (2012), 267404. 